\title{
Perspective on dietary isothiocyanates in the prevention, development and treatment of cancer
}

\author{
Herman Yeger ${ }^{1}$, Reza Bayat Mokhtari ${ }^{1,2}$ \\ ${ }^{1}$ The Hospital for Sick Children, Program in Developmental and Stem Cell Biology, Toronto, ON M5G 0A4, Canada. \\ ${ }^{2}$ Department Biomedical \& Molecular Sciences, Botterell Hall Rm 827, Queen's University, Kingston, ON K7L 3N6, Canada.
}

Correspondence to: Prof. Herman Yeger, Department of Paediatric Laboratory Medicine, Program in Developmental and Stem Cell Biology, Peter Gilgan Centre for Research and Learning, SickKids, 686 Bay St, Toronto, ON M5G 0A4, Canada.

E-mail: hermie@sickkids.ca

How to cite this article: Yeger $\mathrm{H}$, Mokhtari RB. Perspective on dietary isothiocyanates in the prevention, development and treatment of cancer. J Cancer Metastasis Treat2020;6:26. http://dx.doi.org/10.20517/2394-4722.2020.61

Received: 16 Jun 2020 First Decision: 10 Jul 2020 Revised: 16 Jul 2020 Accepted: 21 Jul 2020 Published: 7 Aug 2020

Academic Editor: Sanjay Gupta Copy Editor: Cai-Hong Wang Production Editor: Jing Yu

\begin{abstract}
Epidemiological evidence has highlighted the association of specific diets and a lower incidence of cancer. Foremost, the Mediterranean diet provides high levels of polyphenolics and a high consumption of healthier fats, e.g., as from olive oil. In the Mediterranean region the consumption of vegetables is elevated providing a class of compounds, the isothiocyanates (ITCs) as found in the cabbage family. The ITCs have raised great interest for their health benefits over the past few decades. Some of the key ITC compounds, sulforaphane, phenethylisothiocyanate and benzyl isothiocyanate, have been studied in vitro and in vivo and the data support their promise for cancer chemoprevention, as anti-tumor agents, and for chemoprotection of normal tissues and organs. Along with other polyphenolic compounds in the diet, in general, they also possess key anti-inflammatory properties thus satisfying the criteria for compounds that could intervene in cancer initiation and progression. In this review we provide a larger overview of the advantages of including ITCs in the diet as food or as supplements and speculate on what could constitute a valuable therapeutic strategy for improving and sustaining good health and countering cancer disease in humans.
\end{abstract}

Keywords: Mediterranean diet, isothiocyanates, chemoprevention, chemoprotection, anti-tumor, hormesis, mitohormesis, anti-inflammatory 


\section{INTRODUCTION}

The association between diet and cancer incidence have generated great interest over many years when evidence was obtained that specific regional plant based diets portended significantly lower numbers of specific cancers ${ }^{[1]}$ despite confounding factors that should have predicted an incidence closer to other regions. One such association receiving increased attention both for normal health and a lower incidence of cancer is the association with the Mediterranean diet (MedDiet) ${ }^{[2-4]}$. The MedDiet, also lower in calories, saturated fats, and higher in fiber, also has strong benefits for reduction in cardiovascular disease and generally, inflammatory conditions ${ }^{[3,5]}$. A dissection of the diets in this region highlights the intake of polyphenols and in particular the family of cruciferous vegetables (the Brassica cabbage related family) consumed. The Brassica family contains high amounts of a family of precursors and derivatives of isothiocyanates (ITCs). Populations in the region bordering the Mediterranean also consume large amounts of extra virgin olive oil (EVOO) with a high content of unsaturated fatty acids ${ }^{[4]}$. Interestingly, although a high amount of oleic acid appears to promote cancer growth, the other components in EVOO (hydroxytyrosol, oleuropein, pinoresinol, apigenin, squalene, and maslinic acid) counter the effects of oleic acid and are indeed protective ${ }^{[4]}$. Many other studies support the protective effect of olive oil on gut and the development of colorectal cancer ${ }^{[6,7]}$. Our older studies demonstrated that squalene protects bone marrow progenitors against cancer cytotoxic cisplatin, and that apigenin (along with other flavonoids) can significantly inhibit proliferation of neuroblastoma tumor cells ${ }^{[8]}$. Recent studies indicate that the high content of squalene epoxidase in cancers (in the cholesterol oxidation pathway that metabolizes cytotoxic squalene) and is associated with worse overall survival ${ }^{[9]}$. Thus, although squalene is cytotoxic, its antiinflammatory activity, and the complex of factors in olive oil together operate positively. Thus protection against cancer rests on a complex of dietary factors in the context of human lifestyle habits and genetic contributions.

In this review we present a broader window on the ITC based family of compounds, including our research on sulforaphane (SFN), and make a case for the value of such phytochemicals in cancer prevention and treatment, as well as for other important physiological benefits that together support why ITC based, and in general phytochemical rich diets, working in concert with other healthful components like olive oil can make a real difference to the overall health of people. The review will cover most of the key issues in this class of ITC compounds and end with insights into potential therapeutic applications.

\section{CANCER RISK}

Cancer remains a major risk to health and longevity. Although, according to the National Cancer Institute $(\mathrm{USA})^{[10,11]}$ the decrease in cancer associated deaths have been decreasing in an encouraging manner, the statistics indicate that cancer still constitutes a significant burden (only second to cardiovascular disease) on health and survival. For example, in 2018, approximately 1,735,350 new cases of cancer were estimated to be diagnosed in the United States, and 609,640 people estimated to die from the disease. By 2020, 1,806,590 new cancer cases and 606,520 cancer deaths have been projected. Relative to older data the drop in death rate is trend setting and reflects earlier detection and better treatments. The most common cancers (listed in descending order according to estimated new cases in 2018) are breast cancer, lung and bronchus cancer, prostate cancer, colon and rectum cancer, melanoma of the skin, bladder cancer, non-Hodgkin lymphoma, kidney and renal pelvis cancer, endometrial cancer, leukemia, pancreatic cancer, thyroid cancer, and liver cancer. From the data the number of new cases of cancer (cancer incidence) is 439.2 per 100,000 men and women per year (based on 2011-2015 cases). The number of cancer deaths (cancer mortality) is 163.5 per 100,000 men and women per year (based on 2011-2015 deaths). Cancer mortality is higher among men than women (196.8 per 100,000 men vs. 139.6 per 100,000 women, respectively). When comparing groups based on race/ethnicity and sex, cancer mortality is highest in African American men (239.9 per 100,000) and lowest in Asian/Pacific Islander women (88.3 per 100,000). Estimated national expenditures for cancer 
care in the United States in 2017 were $\$ 147.3$ billion. In future years, costs are likely to increase as the population ages and cancer prevalence increases. Costs are also likely to increase as new, and often more expensive, treatments are adopted as standards of care.

From our perspective, if we then compare with the Canadian cancer statistics ${ }^{[12]}$ we note comparable figures. It is estimated that 1 in 2 Canadians will be diagnosed with cancer in their lifetime, and that 220,400 new cases would be diagnosed in 2019 (113,000 in males and 107, 400 in females). In $2020^{[13]}$, it is projected that 225,800 Canadians will receive a diagnosis of cancer and 83,300 will die of cancer. These estimates are higher than previously reported for 2019, which are expected given the growing and aging population. In contrast, the decline in age-standardized incidence rates (ASIR) overall shows progress is being made for several leading cancers. For example, the ASIRs for some of the commonly diagnosed cancers are declining, reflecting success in cancer-control activities around primary prevention (e.g., tobacco for lung cancer), as well as changes in detection practices (prostate cancer) and possibly screening (colorectal). In addition, there have been dramatic declines in mortality for these cancers, reflecting not only changes in incidence but also improvements in early detection and treatments. In contrast, the lack of progress with pancreatic cancer and the continued increase in the number of cases and deaths expected each year for this and other cancer type shows further efforts in cancer control are urgently required.

Globally speaking, the World Health Organization (IARC, WHO Cancer Facts Sheet, Global Cancer Observatory, March 2019 $9^{[14]}$ ) reported data from 2018 as excerpted for this review: Cancer is the second leading cause of death globally, and is responsible for an estimated 9.6 million deaths in 2018. Globally, about 1 in 6 deaths are due to cancer and approximately $70 \%$ of these occur in low- and middle-income countries. Around one third of deaths from cancer are due to the 5 leading behavioural and dietary risks: high body mass index, low fruit and vegetable intake, lack of physical activity, tobacco use, and alcohol use. Note that the first two indicator risks relate directly to the values inherent in the Mediterranean diet.

As indicated previously the incidence of cancer in a number of favourable areas in the world tends to decrease with better phytochemical enriched diets suggesting that a lot more has to be done in determining how dietary factors can help to bring the incidence sharply down over time and aided by critical changes in lifestyle habits and physiological burdens. Therefore it is vital to try to understand not only how to treat cancer, but importantly how to prevent cancer (ideally early in life) even in light of the driving forces of genetic susceptibility and epigenetics that can play important roles in subverting normal gene expression $^{[15]}$. Finally, it also apparently clear that low- and middle-income populations may not have access to sufficient vegetables and fruits in their diets to meet the threshold amount needed for chemoprevention and chemoprotection. In fact, low-income populations because of limited resources do not purchase the healthier fruits and vegetables they would need, thereby significantly limiting the intake of specific nutrients that could effectively prevent cancer, or as we see, also capable of limiting infections, additional contributing vectors. The MedDiet, very much aligned with other diets that favor greater ingestion of vegetables and fruits, may be circumvented by the trend in the lower economic strata towards poorer typical western diets that favor starches and meats.

On this point the MedDiet has been described with four sustainable benefits ${ }^{[16,17]}$ in terms of nutrition and health, environment, economy, and society and culture. Fresán et al. ${ }^{[18]}$ present a detailed analysis comparing the western diet, MedDiet and provegetarian diet in terms of sustainability, health benefits and cost. The MedDiet had the greatest health benefits but was considered more costly than the others. In a recent review Truzzi et al. ${ }^{[19]}$ examined the broader benefits of sustainability, resilience and health of the MedDiet and indicate that this diet promotes social interaction, protects biodiversity, and ensures conservation and development of traditional activities with a definite emphasis on disease prevention. Given these advantages, for the MedDiet to be transferable to other countries will require a multitude of 
changes in dietary habits ${ }^{[20]}$. In order to translate the MedDiet to a multiethnic population, for example in Australia, there is a need for a consistent definition of the MedDiet to ensure the key elements are captured and translated so that the translated MedDiet model retains authenticity ${ }^{[21]}$. Translating the MedDiet to non-Mediterranean populations with different cultural food habits and cultural customs has been discussed as rather challenging ${ }^{[22]}$.

On the other hand, there are other diets that are also more plant based and can be equally effective and advantageous like the Jiangnan diet in China ${ }^{[23]}$ and the Japanese traditional (Washoku) diet characterized by the high consumption of fish and soybean products and low consumption of animal fat and meat. This diet uses the umami taste to enhance palatability ${ }^{[24]}$. As vegetarian and vegan diets appear to be rising in popularity one might envision a positive reversal in dietary trends going forward. However, as changing dietary habits tends to be difficult to adapt in many ways we stress the necessity to identify key dietary derived compounds that could be easily and cheaply integrated as inexpensive supplements into the most basic of diets.

\section{THE DIETARY CRUCIFEROUS FAMILY OF ITCS}

The cruciferous family of plants, that provide ITCs, includes the brassica species (cabbage family) garden cress, watercress, radish, white mustard and papaya. This class of compounds has been investigated for anti-carcinogenic, chemoprotectant, chemopreventive, and anti-cancer activities, in vitro and in animal models, over the past several decades. A number of excellent detailed reviews and perspectives have been published $^{[25-30]}$. Key members of the ITC family found in the diet, SFN, phenethylisothiocyanate (PEITC), and benzyl isothiocyanate (BITC), show antitumor efficacy, perhaps selectively, against a variety of different cancers $^{[31]}$ and thus have received the greatest attention. These authors have reviewed the molecular mechanisms for individual ITCs and emphasized roles for nuclear factor (erythroid-derived 2)-like 2, (Nrf2) and nuclear factor-kappa beta modulation. In addition, another common and widely studied ITC, allylisothiocyanate (AITC) also possesses anti-inflammatory and antioxidant properties that can improve oxidative stress in pathological processes ${ }^{[32]}$. Singh et al. ${ }^{[30]}$ reviewed the key features of the ITC, erucin (4-methylthio butyl isothiocyanate; 4-MTB-ITC) closely related to SFN, and studied in rodent models of cancer, showing mechanisms of chemopreventive and anticancer effects including apoptosis and cell-cycle arrest, and also enhancing the phase II metabolizing enzymes, suppressing phase I metabolizing enzymes, with enhancement of $\mathrm{Nrf}-2$ that leads to enhanced antioxidant activity.

The known basic structured compound of the ITC family, SFN, derived through digestion or other means, has been well studied and demonstrated by many groups to possess chemopreventive and ant-tumor activities ${ }^{[25,29,32-36]}$. The association with cancer chemoprevention has also been established in animal models and minimally yet in humans ${ }^{[27]}$. Metabolic phenotypes can have a modulatory role $e^{[3]}$. For example, the glutathione-S-transferase T1 phenotype in particular appears to be relevant in how humans physiologically respond to ITCs. Not surprisingly, this speaks to the concept of metabolizer phenotypes for many metabolites (and drugs) in the human population ${ }^{[37]}$. Nevertheless, dissecting the mechanism of action of the different ITCs has become an important area of investigation and their negative effects on several key cancer survival and growth pathways have been documented showing commonalities ${ }^{[25]}$. SFN itself has been identified as a prime example of a compound that triggers the drug detoxification Nrf2-Keap 1 (Kelch-like ECH-associated protein 1) pathway in cells ${ }^{[38,39]}$. As a result there is also the caveat that the SFN induced Nrf2 pathway could protect tumor cells against cytotoxic chemotherapeutics ${ }^{[39]}$. On the other hand, there are several examples of drug potentiation published. Addition of SFN to taxanes enhanced the antitumor efficacy against triple negative breast cancer ${ }^{[40]}$. SFN can synergize with cisplatin to suppress human ovarian cancer ${ }^{[4]}$, and as a component with other anti-tumor phytochemicals ${ }^{[42]}$. Studies from our lab have noted the potent anticancer effects of SFN for both pediatric and adult cancers ${ }^{[35,43,44]}$. Our approach for therapeutic targeting of several cancers (bronchial carcinoids, bladder cancer, and neuroblastoma) was 
to exploit the anti-tumor properties of SFN in combination with targeting of carbonic anhydrase (CA) with acetazolamide, a pan CA inhibitor to perturb the $\mathrm{pH}$ regulation necessary for metastatic progression. Results in vitro and in xenograft models showed a potent anti-tumor inhibition of growth and survival. Whether this combination could similarly inhibit other cancers has yet to be explored. Our studies also raised the possibility that the Nrf2-Keap1 pathway might actually be functionally deficient in certain cancers thereby negating the presumed cancer chemoprotection effect ${ }^{[35]}$. Whether this happens to be the more general case in cancer patients still has to be determined but the low plasma concentrations exhibited by phytochemicals may actually limit or negate tumor cell chemoprotection. An additional interesting aspect revolves around whether Nrf2 activating compounds like SFN could target senescent cancer cells, a potential nidus for metastasis ${ }^{[45]}$. Malavolta et al. ${ }^{[45]}$ studied $15 \mathrm{Nrf2}$ activating compounds including ITCs in the context of adjuvant therapy since these could display senolytic activity.

The growing evidence for ITCs as potent anticancer agents has placed greater recognition on SFN (e.g., being prevalent in broccoli and cabbage, and highly consumed) but it invites speculation that the MedDiet might be advantageous since a number of potent anticancer agents could act cooperatively in all phases of the neoplastic process. It should be noted that a complexity of ITCs are ingested in the MedDiet or other phytochemical diets. The question in terms of cancer prevention is whether dietary factors such as these are introduced in sufficient amounts during development of the fetus, as sourced from the mother, or only appear later in life in the diet, to be maximally beneficial. Phytochemicals with epigenetic modifier properties, such as ITCs, could help to counter environmental toxins exposed to the developing fetus and prenatally ${ }^{[46]}$. There is indeed the caveat that this ITC family of dietary plants, for various reasons (e.g., bitterness, pungency), is often voiced as not favored by or agreeing with children and even adults due to digestive disturbances ${ }^{[47]}$. However, it should be recognized that other, less known members of the cruciferous family are indeed popular and are regularly consumed at higher amounts, for example, in diets in Asia (in cooked form) ${ }^{[48]}$. Thus one would need to determine how specific ITCs could be otherwise administered to achieve the greatest therapeutic benefit.

The essential information on ITCs can be viewed as follows. The three key ITCs, SFN, PEITC, and BITC, are generated from glucosinolates (b-thioglucoside $\mathrm{N}$-hydroxysulfates) by the enzyme myrosinase that is located on the external surface of plant cellsthat becomesactivated and is exposed to the substrates after mastication and cooking or insect damage. ITCs are particularly active via thiocarbamoylation ${ }^{[49]}$. Many phytochemicals exhibit both anti-oxidant and pro-oxidant activities dependent on dose and thus phytochemicals via reactive oxygen species (ROS) induction and in combination with chemotherapeutics compromises tumor cell survival or can be chemopreventive ${ }^{[50]}$. In addition, ITCs like PEITC can tie up glutathione (GSH) thereby raising ROS levels that can compromise tumor cell survival and in particular cancer stem cells $(\mathrm{CSCs})^{[51]}$. Liskova et al. ${ }^{[52]}$ having gleaned the literature emphasize the significant anti-cancer effects of dietary phytochemicals on CSCs in a wide range of cancer types via influencing multiple signaling mechanisms. Targeting of CSCs speaks to the ability to suppress tumor progression from first initiation through malignant progression towards metastasis. Elimination of CSCs or tumor initiating cells would therefore constitute the ideal targets in chemoprevention and anti-tumor targeting during progression. There are documented studies showing targeting of tumor stem cells with good evidence $^{[38,40,43,53]}$. Furthermore, considering all the hallmark features of cancer originally elaborated by Hanahan and Weinberg ${ }^{[54]}$ and now reviewed and expanded on by Girotti et al. ${ }^{[55]}$, who describe how galectins can affect tumor progression, it is obvious that ITCs can have a significant impact on all of these, including galectins ${ }^{[56]}$. Thus it is not surprising that there is a building literature on the cancer chemoprevention and anti-tumor progression by ITCs.

ITCs are metabolized in the liver by the mercapturic acid pathway and thereafter eliminated in the urine being detected after consumption of glucosinolates. From the perspective of elimination it is interesting to 
note that ITCs are promising chemopreventives for bladder cancer $^{[57]}$. In other words, routes of metabolism and elimination may be key sites for their biological activity in certain cancers. Aside from these 3 main natural compounds, more than 20 natural and synthetic ITCs have been studied for their anti-carcinogenic potential and properties ${ }^{[25,58,59]}$. In clinical trials conducted or in progress on a variety of cancers, ITCs are showing positive outcomes on different biological parameter ${ }^{[58]}$. Importantly the efficacy has varied considerably dependent upon the test system, target tissues, type of carcinogen involved, the particular ITC, dose, and dosing regimen. In a limited series of experiments in humans, activation of a carcinogen was blocked supporting the idea of chemoprevention. High performance liquid chromatography has afforded a reliable means of measuring ITC levels and kinetics.

From the perspective that ITCs might be consumed during cancer therapy, and in trying to understand normal tissue/organ chemoprotection, the following biochemical pathway has received great attention. The ITCs are potent inducers of the phase 2 enzymes, the Nrf2-Keap1 pathway mentioned above, involved in detoxification of carcinogens, mutagens and a larger variety of toxins constituting the protective system in cells, both normal and cancerous ${ }^{[39]}$. However, there is thinking that this complexly regulated pathway ${ }^{[60]}$, being upregulated by ITCs, may counter chemotherapeutic efficacy compromising the therapeutic outcome. On the other hand, the evidence that ITCs can potentiate the efficacy of chemotherapeutics ${ }^{[40]}$, their rapid metabolism and elimination may actually interfere with the Nrf2 pathway based detoxification scenario.

It is well recognized and known, that tumor cells can invoke other mechanisms of drug resistance of greater impact. Also consider that ITCs have reliable documented broad effects on cell cycle progression, proliferation, apoptosis, and multiple cell survival signaling pathways supporting interference in tumor progression in many cancers and in a dose dependent manner. Concomitantly, the induction of phase 2 enzymes by ITCs may also play a significant role in protecting normal tissues against cytotoxics ${ }^{[39]}$.

Epigenetics play a significant role in cancer. Relevant to epigenetic regulation, ITCs are weaker but still effective inhibitors of histone deacetylases, histone deacetylases ${ }^{[35]}$, and although changes in histone acetylation may be minimal, some of their potency may involve modulation of cell activity epigenetically ${ }^{[6]]}$. In fact, combinations of SFN (and likely other ITCs) with other phytochemicals (e.g., genistein) could operate with enhanced epigenetic activity ${ }^{[62,63]}$ and via mechanisms involving inhibition of non-coding RNAs (e.g., miRNAs) ${ }^{[64]}$. Since epigenetic therapy inhibits metastases by disruption of the metastatic niche, i.e., the tumor microenvironment ${ }^{[65]}$, ITCs may be advantageous at multiple steps in the malignant process. In skin, evidence exists that ITCs can normalize epigenetic marks altered during tumorigenesis while inhibiting melanoma growth and survival ${ }^{[66]}$. PEITC also modulates epigenetic writers and erasers to restrict tumor development ${ }^{[5]}$. Regulation of the epigenetic machinery may be a major function of ITCs in chemoprevention and perhaps chemoprotection of normal tissues. Whether the mechanism involves favorably rebalancing miRNAs is still to be determined.

Interestingly, ITCs target mitochondria and the electron transport chain to provoke cancer cell-selective death programming. ITCs incur mitochondrial disruption of electron transport generation to trigger apoptosis as chemopreventives ${ }^{[67]}$. The mechanism here for SFN is thought to be targeting critical mitochondrial cysteine residues in complex III but also complex I-II by SFN; it should be noted that ITCs (with the highly reactive $\mathrm{R}-\mathrm{N}=\mathrm{C}=\mathrm{S}$ moiety) in general are reactive with accessible cysteine residues in proteins, thus possibly able to inactivate multiple key signaling proteins favoring cancer growth and survival ${ }^{[68]}$. As a primary example, SFN suppresses the growth of the very aggressive glioblastoma tumor cells, the stem-cell like spheroids (the CD133 fraction) and xenografts through multiple signaling pathways, with a lessor effect on normal brain cells ${ }^{[69]}$. This study also demonstrated it can cross the bloodbrain barrier and so may have versatility in enhancing other therapeutics. Despite this ability our own xenograft studies did not show any toxicity to the mice and obviously higher consumption of cruciferous 
vegetables have not revealed any cytotoxicity likely due to the more rapid elimination and limiting plasma concentrations of such compounds.

On the other hand, along a cautionary line, the clinical value of ITCs as chemopreventives and anticancer agents has been tempered by evidence that immune cell functions ( $\mathrm{T}$ cell and NK cells) may be compromised even at low doses (i.e., reducing proliferation of peripheral blood mononuclear cells) ${ }^{[27]}$. The real question is whether ITCs access these cell compartments in vivo during ingestion and whether rapid conjugation (e.g., by GSH) yields derivatives that are less active against immune cells but are still potent chemopreventives. Since ITCs and metabolites are present in urine and plasma in the nanogram range and rapidly eliminated ${ }^{[70]}$ it is conceivable that this very low plasma level alone counters any deleterious effects, but still permits chemoprevention and anti-tumor effects in a selective uptake and concentration dependent manner. Certainly, as in vitro experiments are not confounded in this way, does this indicate that a deleterious effect is yet possible on the immune system? Noteworthy is that ingestion of large amounts of ITCs has not been found to compromise human health likely due to extensive elimination keeping plasma concentrations $\operatorname{low}^{[58]}$. In this context, even low concentrations may in fact be physiologically active since hormetic effects (see below) and routes of metabolism and tissue accumulation may determine the ultimate efficacy.

It is important to put such observations in the context of diets where cruciferous plants are consumed on a regular basis without any obvious negative outcomes. In fact, epidemiological observations vis-à-vis the MedDiet generally suggests the opposite. Furthermore, plant based diets comprise a very large array of different phytochemicals that can act additively, synergistically or antagonistically or can protect against negative effects ${ }^{[59]}$. As examples of synergistic effects between ITCs and other polyphenolics in terms of anti-inflammatory efficacy a number of papers have been published ${ }^{[71,72]}$. ITCs in combination with antioxidants such as selenium and polyphenolics such as genistein can operate at an epigenetic level ${ }^{[7,74]}$. The ITCs AITC and SFN are synergistic for chemoprevention of non-small cell lung carcinoma ${ }^{[75]}$. More examples exist, however this area of research is still not mainstream as many scientists have not yet appreciated the larger therapeutic efficacy of the vast complexity of functional dietary factors.

Overall, epidemiological observations favor the beneficial side of ingesting cruciferous plants along with many other unrelated positively influential dietary components (e.g., as found in olive oil). Jaman and Sayeed $^{[76]}$ reviewed evidence for ellagic acid, sulforaphane, and ursolic acid as possible therapy for breast cancer, and although suggestive positive overall, they raised questions about compounds that could negatively affect both tumor cells and normal cells. As mentioned before, dose is a critical parameter, and as plasma concentrations of phytochemicals tend to be self limiting in vivo and phytochemicals often rapidly inactivated or eliminated ${ }^{[58,70]}$, one needs to consider that most phytochemicals would likely behave within the parameters and kinetics of hormesis ${ }^{[77]}$.

\section{HORMETIC EFFECTS MAY GOVERN THE EFFICACY OF PHYTOCHEMICALS AND LIMIT TOXICITY}

Hormesis, the low dose stress modulatory effect, describes how phytochemicals (including many other agents) are bimodal in their function often acting positively at a significantly lower dose and acting negatively at higher doses ${ }^{[78-81]}$. There is the idea that early life stressors can lead to resilience in later life ${ }^{[78]}$, and if thought of in the context of dietary factors then early exposure to chemopreventive compounds might condition the body for greater resilience or make it refractory to oncogenic stimuli ${ }^{[80]}$. At another cellular level, acknowledging that mitochondria are absolutely essential for life, mitohormesis (an adaptive stress response) defines a biological response where reduction in mitochondrial stress can lead to significant increments in health and vitality proscribing what is felt to be the anti-aging paradigm ${ }^{[3,82]}$. Mediators of 
mitohormesis such as FGF21 and GDF15 have been described vis-à-vis increased lifespan ${ }^{[82]}$. It is also proposed that altered mitohormesis primes a subpopulation of cancer cells to upregulate mitochondrial stress response leading to an adaptive metastatic phenotyp $\mathrm{e}^{[83]}$. Therefore, theoretically, phytochemicals that have benefits on normal organ function via support of healthy mitochondria may counter cancer altering mitohormesis.

Thus the working concept here, capitalizing on the precepts of hormesis, is that for phytochemicals to be effective as chemopreventives at the earliest stages and continuously as guardians against tumorigenesis, combinations must be sought that work within the low dose range permitting adaptive responses and not toxicological responses ${ }^{[77]}$. This would then provide the protection against biological stresses affecting normal homeostasis of tissues and cells and that can intervene in tumorigenesis, whether genetic or epigenetic. To paraphrase the summary statements in the review article on stress response by Zelenka et al. ${ }^{[84]}$ they indicate the following. Upregulation of the cellular stress response pathways are governed by exercise, natural compounds, or drugs that protect the body against carcinogenesis. However, the same stress response pathways may protect cancer cells against metabolic and oxidative stress associated with metastatic dissemination and anticancer therapy. In cancer prevention upregulating stress responses of the body are desirable, but anticancer drugs that downregulate stress response in tumor cells have just emerged. In targeting of the stress response, less specific multi-targeting approaches are often more successful than those targeting a single molecular target. It is noteworthy in stress response research that the dose of the stressor and the time to adaptation dramatically influence the outcome of each study.

Of relevance here is the paradox of hormesis with the low-dose beneficial effects of stressors, viewing nearly all phytochemicals as stressors, but having an intrinsic high dose toxic effect. Thus combinations of phytochemicals, if well selected, when operating at low doses could maintain a precious homeostatic state supporting normal functions and the anti-neoplastic resistant state in all tissues and organs. Biological systems are innately adaptive to stressors and respond ideally if phytochemicals are delivered with optimum duration and exposure.

\section{INFLAMMATION AS A CRITICAL TARGET OF THE CANCER PROCESS}

There is consensus amongst most scientists and physicians that inflammation is a critical component of many pathological processes. For example, Tsoupras et al. ${ }^{[85]}$ analyzing the driving force behind cardiovascular disease identified systemic inflammation and not cholesterol as the culprit. Especially in cancer disease progression the inflammatory microenvironment can drive the malignant phenotype ${ }^{[86]}$. In fact, any perturbation in normal homeostasis is announced to the immune regulatory system in the body and invites inspection and reaction from a variety of immune cells orchestrated to attempt resolution. In this way it is well recognized that local or systemic inflammation can play a major role in how the cancerous process rolls out ${ }^{[86]}$. In the oncogenic microenvironment, as a perturbation of normal tissue homeostasis, inflammatory events figure early on in the pathologic process and exacerbate the damage by humoral factors and through recruitment of immune cells ${ }^{[86]}$. This is where the anti-inflammatory activity of phytochemicals and especially ITCs has relevance.

The ITCs and other phytochemicals could have a major chemoprevention impact on the development of cancer precursor lesions, whether as occurring in embryonal derived cancers, for example, Wilms tumor $^{[87,88]}$ or on in situ precursor lesions well documented in adult cancers ${ }^{[74]}$. Certainly, the incidence of precursor lesions is significantly higher than the progression to frank cancer ${ }^{[89]}$. The derivation of premalignant lesions can be understood in the context of the theoretical bases of the two hit hypothesis, initiation and promotion, and the current day elaboration in genetic and epigenetic term ${ }^{[6,54]}$. Furthermore, we now have a much greater understanding of tumor progression towards metastasis, whether incurred locally or via circulating tumor cells ${ }^{[13,90]}$; all should be amenable to dietary phytochemicals. Therefore 
chemoprevention has a true functional basis; however, thus far scientists have not been able to propose a universal working strategy for implementation of cancer chemoprevention.

In addition, the tumor cell (precursor state or subsequently) microenvironment plays a significant role in how the malignant process evolves and where and how one central player, the cancer associated fibroblasts (CAFs), play a major role ${ }^{[91]}$. Thus in this microenvironment of a developing cancer and thereafter during tumor progression (with increasing epigenetic aberrations) towards a metastatic phenotype, immune surveillance is involved and immune responses are elicited and manipulated ${ }^{[92]}$. There is a growing understanding that CAFs play a key role in immunosurveillance in the tumor microenvironment. In parallel, and even earlier, innate immune responses elicited from the surrounding normal cells would cause release of inflammatory mediators necessary for the full- blown immune response ${ }^{[93]}$. However, in the resulting complex of immune cells both inhibitors and checkpoints are produced and can suppress innate immunity. One can therefore surmise that endogenous anti-inflammatory mediators or exogenously provided as phytochemicals may help modulate the immune response in a positive fashion to thwart malignant progression and permit rapid return to homeostasis.

In this regard advice has yet to emerge on an appropriate diet that could accurately provide the necessary dietary phytochemicals that might prove most effective in blocking cancer initiation and progression and are intrinsically anti-inflammatory ${ }^{[94-97]}$. Many phytochemicals possess these properties and a routine intake of the right combinations of phytochemicals that would include the ITCs, well recognized for their anti-inflammatory activity, might fit the bill. In addition, and for example, as a potential combination phytochemical, curcumin stands out in this regard ${ }^{[98]}$. Combinations of phytochemicals, selected appropriately, would have a greater inhibitory impact on inflammation, preventively or during progressive states to limit or inhibit the evolution of a cancer and also thereby the damage to normal tissues ${ }^{[94,99]}$. This has been the working hypothesis favoring the MedDiet. Of concern, as voiced by Tsoupras et al. ${ }^{[85]}$ is that, if inflammation fails to resolve, due to persistence of a triggering event or unsuccessful block, then chronic inflammation may exacerbate the damaging outcome. This is where a continuous exposure to anti-inflammatory phytochemicals could rebalance the inflammatory state. In tumor progression, tumor cells are able to recruit immune cells to promote tumor progression to a higher state of malignancy and metastasis $^{[13]}$. Thus it will be necessary to select phytochemicals, and also other natural compounds, that are not only potent anti-inflammatories but also anti-metastatic. In addition, combinations of antiinflammatory phytochemicals would likely act additively or synergistically to enhance the positive outcome. In terms of biomarkers that could identify cancer prevention potential it has been noted that a pro-coagulative state is related to not only increased risk of cardiovascular diseases but also certain adult cancers. Grafetstätter et al. ${ }^{[100]}$ assessed lower alcohol and meat consumption population studies that highlight thrombomodulin and thrombopoietin as two markers that quantitatively associate with chronic disease development. Thus other biomarkers should be sought that can predict ongoing biological processes underlying cancer development.

\section{THERAPEUTIC STRATEGY}

The cruciferous family of dietary plants provides ITCs, and historically such plants have been used for medicinals, cooking, and in massage oils from their origins in different members of the cruciferous family. In addition to these attributes, as an example, benzyl ITC, has shown bacteriostatic, bacteriocidal and anthelmintic and vermifuge properties at low concentrations. Uppal et al. ${ }^{[101]}$ discuss this but raised the caveat of poor bioavailability for a number of intrinsic chemical properties ${ }^{[68]}$. Uppal et al ${ }^{[101]}$ then showed that within a complex of chitosan nanoparticles as carriers there is significantly improved efficacy. In fact, bio-nanocarriers have proven highly advantageous for efficient delivery of other promising phytochemical therapeutics such as curcumin ${ }^{[102]}$. Efforts are being made for improving the bioavailability of ITCs $^{[103]}$. 
Keeping hormetic effects in mind, and the fact that such compounds can accumulate in cells and tissues, would bioavailability still remain a true physiological limitation?

As with many phytochemicals ingested daily over a lifetime, maintenance of a lower plasma concentration and eliminating excess, may be of real benefit since ITCs are also beneficial for normal organ functions as fully evident ${ }^{[60]}$. What is of concern is whether humans would maintain dietary practices that could provide the necessary amounts of phytochemicals, like the ITCs, to serve for chemoprevention and chemoprotection. Furthermore, as the evidence for additive and synergistic positive effects amongst a variety of phytochemicals is compelling ${ }^{[70]}$, one would hope that ideal combinations of phytochemicals could be uncovered to provide supplements that could be included in products acceptable to the general populations. One could envision incorporating such combinations in everyday desirable foods sought by most.

Finally, keeping an open mind, one might envision combining ITCs with other natural related compounds, e.g., curcumin, showing potent anti-tumor and anti-inflammatory activity as well as having positive physiological effects that could provide a better chemoprotective environment. In addition, in light of the metabolic reprogramming of the cancer microenvironment ${ }^{[104]}$ the widely used drug for treatment of type 2 diabetes (T2D), metformin, a biguinide, derived almost a century ago from parent compound galengin found in French lilac, has shown anti-tumor effects perturbing glucose metabolism in tumor cells ${ }^{[105]}$. When considering that hormesis also includes mitohormesis perhaps adding metformin (or a phytochemical equivalent) to the ITCs regimen could prove more effect in targeting tumor evolution or progression in the earliest stages. This of course is only speculative but it does invite supporting further research on this subject. Another factor is that if ITCs from food have limiting bioavailability/absorption could this be balanced out by the beneficial kinetics of hormesis? Since many phytochemicals operate with the kinetics of hormesis, in order to keep the dosage within the positive optimal low dose range, it may be necessary to obtain necessary pharmacokinetic information from acute, intermittent and chronic administration (i.e., ingestion). One might surmise that this strategy would not constitute a burden on lifestyle as many people have their morning coffee (or tea, both with touted health benefits) with little thought to daily frequency. Many folks are also cognizant of the antioxidant value of phytochemicals, however it should also be recognized that phytochemicals operate as anti-cancer agents through multiple mechanisms ${ }^{[106]}$.

It would bode well for all global communities to re-examine their diets and realign them more effectively with a more uniform country-wide distribution of foods that could attain the goal of improving health in concert with increased longevity. As discussed before many regions of the world can identify diets that constitute wholly or partially the basic components and endorse appropriate supplementations in a cost effective form containing the ITCs, polyphenolics, and healthy fats found in vegetables and fruits. Therefore, the MedDiet may emerge as a medical prescription able to win against unhealthy eating habits from other areas of the globe ${ }^{[107]}$. A major global challenge is overriding the wave of obesity in many cultures $^{[108]}$ with links to the growing rise in $\mathrm{T} 2 \mathrm{D}^{[109]}$. Therefore it is reasonable to raise the progressive idea of supplementing reasonably balanced diets with the beneficial compounds found in the MedDiet, when bulk produced to lower costs, and deriving the optimal concentrations. Adding an optimal dose of chemoprevention/chemoprotection to the morning coffee or tea, if pre-formulated and not altering taste, etc., would not raise any concerns if proven beneficial. Addressing this aspect Yagishita et al. ${ }^{[110]}$ reviewed the current state of knowledge regarding the different aspects of formulations, bioavailability and efficacy and what might be needed to implement a public health paradigm for global application. Quirante-Moya et al. ${ }^{[11]}$ also question whether bioactive compounds from Brassica species are being studied in the right way given the lack of solid clinical trial data thus far. Moreover, ITC containing plant species are present in cold and hot climates thus permitting global acquisition and evaluation. 


\section{Cancer initiation}

Cancer metastasis

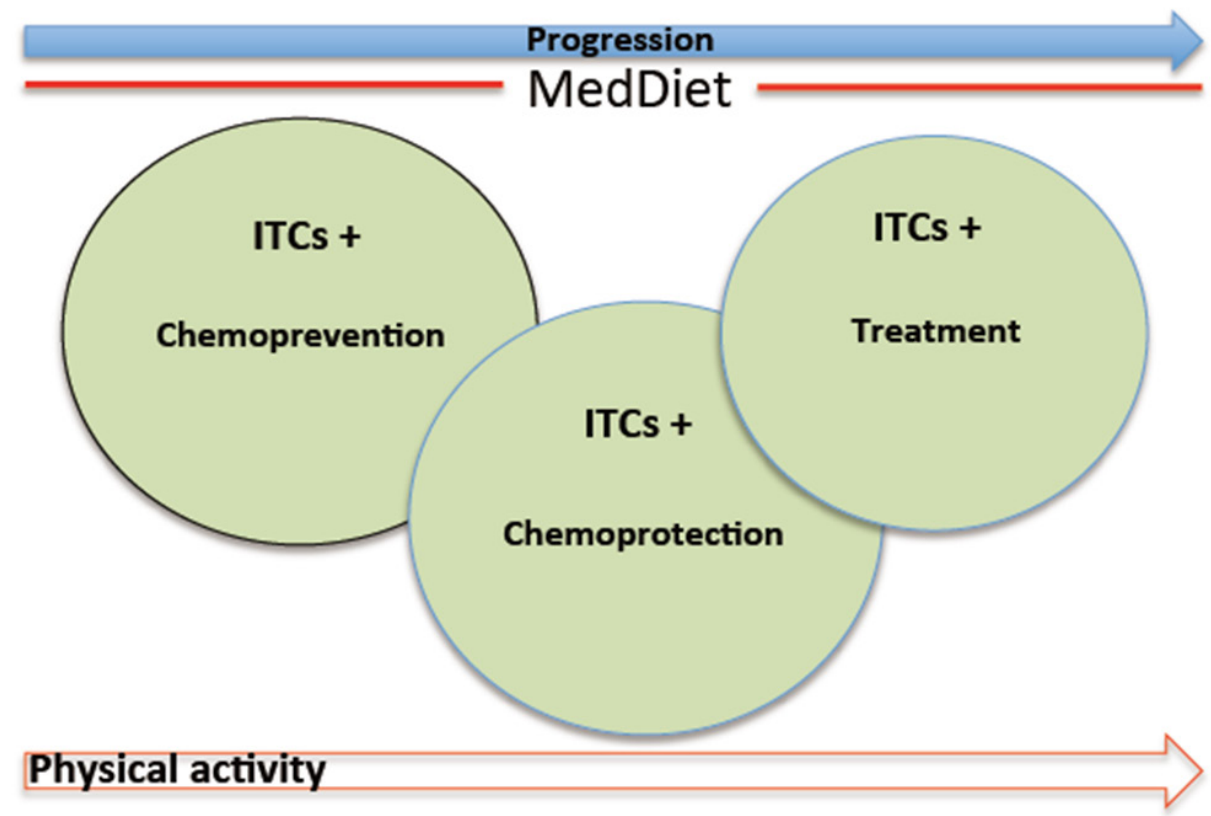

Figure 1. Schema depicts how diets, like the Mediterranean diet (MedDiet), as part of a healthy lifestyle can contribute sustainably and positively to the health of humans, the chemoprevention of cancer, inhibition of tumor progression and metastasis, and for the treatment of cancers. Concomitantly the therapeutic components in the MedDiet can also chemoprotect normal tissues and organs. All these effects are mediated by the isothiocyanates (ITCs), other phytochemicals, and oils present in this diet. ITCs have also shown potentiation of other chemotherapeutic agents, thus expanding their versatility. Maintaining good physical activity running in parallel with a chemoprotective diet can also play a significant role in ameliorating the adverse effects of T2D, obesity and cardiac disease which can exacerbate the deleterious effects of cancer

Thus it is time we view chemoprotection, chemoprevention and hormesis governed homeostasis, as a potential advantage of ITCs and other phytochemicals, and a necessary strategy for well being and a healthier, longer life $\mathrm{e}^{[3,58]}$. From this viewpoint a lot more can be done to bring anti-cancer and health promoting phytochemicals into global diets.

\section{CONCLUSION}

Here we have tried to present a new supportive strategy for preventing the onset and the treatment of cancer if attempting to intervene optimally, and if possible, for effectively targeting the entire tumorigenic process especially malignant progression. There are numerous phytochemicals with potential for cancer prevention including ITCs ${ }^{[26]}$. Concomitantly we indicate that inclusion of an optimal balance of phytochemicals could also protect normal organs. How these phytochemicals would be selected and cognizant of their interactions has been extensively discussed ${ }^{[112]}$; however it will require more studies to determine how ITCs interact with other phytochemicals and if there are effects on bioavailability and function. As a side benefit to the inclusion of ITCs the anti-microbial activity of ITCs ${ }^{[113]}$ would add an important further element towards achievement of a healthier lifespan.

Undoubtedly, the ultimate goals of preventing and curing cancer are perhaps very lofty at present. At the very least, as stated by others, cancer could be relegated to the status of a non life-threatening disease kept suppressed and sub-clinical. It may turn out, given the complexity of determining the ideal chemopreventive and anti-tumor diets or treatments, that ITCs in combination with other phytochemicals might be capable of controlling cancer with this objective in mind. As a last thought on the subject, a recent article by Pedersen in The Scientist ${ }^{[114]}$ presented a convincing review of evidence for exercise in prevention 
and significantly retarding malignant progression of cancer while Musci et al. ${ }^{[115]}$ voiced the same message for maintenance of skeletal muscle and healthspan extension via exercise induced balanced mitohormesis. Given that ITCs could positively influence mitochondrial functions, bolstering mitohormesis, and that cancer cells and normal cells differ sufficiently in mitochondrial functions, ITCs and other phytochemicals could show the desirable selectivity.

Figure 1 schema presents a common sense strategy illustrating that leading a physically active lifestyle and consuming a phytochemical rich MedDiet, where ITCs figure prominently, may be the best defense against cancer and concomitantly for maintaining the health of all organ systems.

\section{DECLARATIONS}

\section{Authors' contributions}

Contributed equally to the literature search and the writing of this manuscript: Yeger H, Mokhtari RB

First developed the concept and the Figure Schema: Yeger H

\section{Availability of data and materials}

Not applicable.

\section{Financial support and sponsorship}

None.

\section{Conflicts of interest}

Both authors declared that there are no conflicts of interest.

\section{Ethical approval and consent to participate}

Not applicable.

\section{Consent for publication}

Not applicable.

\section{Copyright}

(c) The Author(s) 2020.

\section{REFERENCES}

1. Hidalgo-Mora JJ, García-Vigara A, Sánchez-Sánchez ML, García-Pérez MÁ, Tarín J, et al. The Mediterranean diet: a historical perspective on food for health. Maturitas 2020;132:65-9.

2. La Vecchia C. Mediterranean diet and cancer. Public Health Nutr 2004;7:965-8.

3. Di Daniele N, Noce A, Vidiri MF, Moriconi E, Marrone G, et al. Impact of Mediterranean diet on metabolic syndrome, cancer and longevity. Oncotarget 2017;8:8947-79.

4. Mazzocchi A, Leone L, Agostoni C, Pali-Schöll I. The secrets of the Mediterranean diet. Does [only] olive oil matter? Nutrients 2019;11:2941

5. Tuttolomondo A, Simonetta I, Daidone M, Mogavero A, Ortello A, et al. Metabolic and vascular effect of the mediterranean diet. Int J Mol Sci 2019;20:4716.

6. Borzì AM, Biondi A, Basile F, Luca S, Vicari ESD, et al. Olive oil effects on colorectal cancer. Nutrients 2018;11:32.

7. Peluso I, Yarla NS, Ambra R, Pastore G, Perry G. MAPK signalling pathway in cancers: olive products as cancer preventiveand therapeutic agents. Sem Cancer Biol 2019;56:185-95.

8. Das B, Antoon R, Tsuchida R, Lotfi S, Morozova O, et al. Squalene selectively protects mouse bone marrow progenityors against cisplatin and carboplatin-induced cytotoxicity in vivo without protecting tumor growth. Neoplasia 2008;10:1105-19.

9. Shen T, Lu Y, Zhang Q. High squalene epoxidase in tumors predicts worse survival in patients with hepatocellular carcinoma: integrated bioinformatic analysis on NAFLD and HCC. Cancer Control 2020;27:1073274820914663.

10. Siegel RL, Miller KD, Jemal A. Cancer statistics, 2019. CA Cancer J Clin 2019;69:7-34.

11. Siegel RL, Miller KD, Jemal A. Cancer statistics, 2020. CA Cancer J Clin 2020;70:7-30. 
12. Public Health Agency of Canada; Statistics Canada; Canadian Cancer Society; provincial/territorial cancer registries. Release notice Canadian cancer statistics 2019. Avis de publication - Statistiques canadiennes sur le cancer 2019. Health Promot Chronic Dis Prev Can 2019;39:255.

13. Brenner DR, Weir HK, Demers AA, Ellison LF, Louzado C, et al; Canadian Cancer Statistics Advisory Committee. Projected estimates of cancer in canada in 2020. CMAJ 2020;192:E199-205.

14. World Health Organization, Newsroom, Facts Sheet, Detail, Cancer. Available from: https://www.who.int/news-room/fact-sheets/detail/ cancer. [Last accessed on 28 Jul 2020]

15. Fares J, Fares MY, Khachfe HH, Salhab HA, Fares Y. Molecular principles of metastasis: a hallmark of cancer revisited. Signal Transduct Target Ther 2020;5:28.

16. Dernini S, Berry EM, Serra-Majem L, La Vecchia C, Capone R, et al. Med Diet 4.0: the Mediterranean diet with four sustainable benefits. Public Health Nutr 2017;20:1322-30.

17. Berry EM. Sustainable food systems and the mediterranean diet. Nutrients 2019;11:2229.

18. Fresán U, Martínez-González MA, Sabaté J, Bes-Rastrollo M. Global sustainability (health, environment and monetary costs) of three dietary patterns: results from a Spanish cohort (the SUN project). BMJ Open 2019;9:e021541.

19. Truzzi ML, Puviani BM, Tripodi A, Toni S, Farinetti A, et al. Mediterranean diet as a model of sustainable, resilient and healthy diet. Prog Nutr 2020;22:388-94.

20. Martínez-González MÁ, Hershey MS, Zazpe I, Trichopoulou A. Transferability of the mediterranean diet to non-mediterranean countries. What is and what is not the mediterranean diet. Nutrients 2017;9:1226.

21. George ES, Kucianski T, Mayr HL, Moschonis G, Tierney AC, et al. A mediterranean diet model in Australia: Strategies for translating the traditional mediterranean diet into a multicultural setting. Nutrients 2018;10:465.

22. Mantzioris E, Villani A. Translation of a mediterranean-style diet into the Australian dietary guidelines: a nutritional, ecological and environmental perspective. Nutrients 2019;11:2507.

23. Wang J, Lin X, Bloomgarden ZT, Ning G. The Jiangnan diet, a healthy diet pattern for Chinese. J Diabetes 2020;12:365-71.

24. San Gabriel A, Ninomiya K, Uneyama $\mathrm{H}$. The role of the japanese traditional diet in healthy and sustainable dietary patterns around the world. Nutrients 2018;10:173.

25. Wu X, Zhou QH, Xu K. Are isothiocyanates potential anti-cancer drugs? Acta Pharmacol Sin 2009;30:501-12.

26. Ranjan A, Ramachandran S, Gupta N, Kaushik I, Wright S, et al. Role of phytochemicals in cancer prevention. Int J Mol Sci 2019;20:4981.

27. Gründemann C, Huber R. Chemoprevention with isothiocyanates - From bench to bedside. Cancer Lett 2018;414: 26-33.

28. Zhang Z, Bergan R, Shannon J, Slatore CG, Bobe G, et al. The role of cruciferous vegetables and isothiocyanates for lung cancer prevention: current status, challenges, and future research directions. Mol Nutr Food Res 2018;62:e1700936.

29. Kamal MM, Akter S, Lin CN, Nazzal S. Sulforaphane as an anticancer molecule: mechanisms of action, synergistic effects, enhancement of drug safety, and delivery systems. Arch Pharm Res 2020;43:371-84.

30. Singh D, Arora R, Bhatia A, Singh H, Singh B, et al. Molecular targets in cancer prevention by 4-(methylthio)butyl isothiocyanate - a comprehensive review. Life Sci 2020;241:117061.

31. Soundararajan P, Kim JS. Anti-carcinogenic glucosinolates in cruciferous vegetables and their antagonistic effects on prevention of cancers. Molecules 2018;23:2983.

32. Mastuo T, Miyata Y, Yuno T, Mukae Y, Otsubo A, et al. Molecular mechanisms of the anti-cancer effects of isothiocyanates from cruciferous vegetables in bladder cancer. Molecules 2020;25:575.

33. Amjad AI, Parikh RA, Appleman LJ, Hahm ER, Singh K, et al. Broccoli-derived sulforaphane and chemoprevention of prostate cancer: from bench to bedside. Curr Pharmacol Rep 2015;1:382-90.

34. Zhang Y, Tang L. Discovery and development of sulforaphane as a cancer chemopreventive phytochemical. Acta Pharmacol Sin 2007;28:1343-54.

35. Bayat Mokhtari R, Baluch N, Homayouni TS, Morgatskaya E, Kumar S, et al. The role of Sulforaphane in cancer chemoprevention and health benefits: a mini-review. J Cell Commun Signal 2018;12:91-101.

36. Sita G, Hrelia P, Graziosi A, Morroni F. Sulforaphane from cruciferous vegetables: recent advances to improve glioblastoma treatment. Nutrients 2018;10:1755.

37. Mayers JR, Vander Heiden MG. Nature and nurture: what determines tumor metabolic phenotypes? Cancer Res 2017;77:3131-4.

38. Kubo E, Chhunchha B, Singh P, Sasaki H, Singh DP. Sulforaphane reactivates cellular antioxidant defense by inducing Nrf2/ARE/Prdx6 activity during aging and oxidative stress. Sci Rep 2017;7:14130.

39. Kitamura H, Motohashi H. NRF2 addiction in cancer cells. Cancer Sci 2018;109:900-11.

40. Burnett JP, Lim G, Li Y, Shah RB, Lim R, et al. Sulforaphane enhances the anticancer activity of taxanes against triple negative breast cancer by killing cancer stem cells. Cancer Lett 2017;394:52-64.

41. Kan SF, Wang J, Sun GX. Sulforaphane regulates apoptosis- and proliferation-related signaling pathways and synergizes with cisplatin to suppress human ovarian cancer. Int J Mol Med 2018;42:2447-58.

42. Negrette-Guzmán M. Combinations of the antioxidants sulforaphane or curcumin and the conventional antineoplastics cisplatin or doxorubicin as prospects for anticancer chemotherapy. Eur J Pharmacol 2019;859:172513.

43. Mokhtari RB, Baluch N, Morgatskaya E, Kumar S, Sparaneo A, et al. Human bronchial carcinoid tumor initiating cells are targeted by the combination of acetazolamide and sulforaphane. BMC Cancer 2019;19:864.

44. Islam SS, Mokhtari RB, Akbari P, Hatina J, Yeger H, et al. Simultaneous targeting of bladder tumor growth, survival, and epithelial- 
to-mesenchymal transition with a novel therapeutic combination of acetazolamide (AZ) and sulforaphane (SFN). Target Oncol 2016;11:209-27.

45. Malavolta M, Bracci M, Santarelli L, Sayeed A, Pierpaoli E, et al. Inducers of Senescence, toxic compounds, and senolytics: the multiple faces of Nrf2-activating phytochemicals in cancer adjuvant therapy. Mediators Inflamm 2018;2018:4159013.

46. Li S, Chen M, Li Y, Tollefsbol TO. Prenatal epigenetics diets play protective roles against environmental pollution. Clin Epigenetics 2019;11:82.

47. Bell L, Oloyede OO, Lignou S, Wagstaff C, Methven L. Taste and flavor perceptions of glucosinolates, isothiocyanates, and related compounds. Mol Nutr Food Res 2018;62:e1700990.

48. Wu QJ, Yang G, Zheng W, Li HL, Gao J, et al. Pre-diagnostic cruciferous vegetables intake and lung cancer survival among Chinese women. Sci Rep 2015;5:10306.

49. Nigro C, Leone A, Fiory F, Prevenzano I, Nicolò A, et al. Dicarbonyl stress at the crossroads of healthy and unhealthy aging. Cells 2019;8:749.

50. Chikara S, Nagaprashantha LD, Singhal J, Horne D, Awasthi S, et al. Oxidative stress and dietary phytochemicals: role in cancer chemoprevention and treatment. Cancer Lett 2018;413:122-34.

51. Lv HH, Zhen CX, Liu JY, Shang P. PEITC triggers multiple forms of cell death by GSH-iron-ROS regulation in K7M2 murine osteosarcoma cells. Acta Pharmacol Sin 2020; doi: 10.1038/s41401-020-0376-8.

52. Liskova A, Kubatka P, Samec M, Zubor P, Mlyncek M, et al. Dietary phytochemicals targeting cancer stem cells. Molecules 2019;24:899.

53. Nguyen YT, Moon JY, Ediriweera MK, Cho SK. Phenethyl isothiocyanate suppresses stemness in the chemo- and radio-resistant triplenegative breast cancer cell line MDA-MB-231/IR via downregulation of metadherin. Cancers (Basel) 2020;12:268.

54. Hanahan D, Weinberg RA. Hallmarks of cancer: the next generation. Cell 2011;144:646-74.

55. Girotti MR, Salatino M, Dalotto-Moreno T, Rabinovich GA. Sweetening the hallmarks of cancer: Galectins as multifunctional mediators of tumor progression. J Exp Med 2020;217:e20182041.

56. Tian H, Zhou Y, Yang G, Geng Y, Wu S, et al. Sulforaphane-cysteine suppresses invasion via downregulation of galectin-1 in human prostate cancer DU145 and PC3 cells. Oncol Rep 2016;36:1361-8.

57. Abbaoui B, Lucas CR, Riedl KM, Clinton SK, Mortazavi A. Cruciferous vegetables, isothiocyanates, and bladder cancer prevention. Mol Nutr Food Res 2018;62:e1800079.

58. Palliyaguru DL, Yuan JM, Kensler TW, Fahey JW. Isothiocyanates: translating the power of plants to people. Mol Nutr Food Res 2018;62:e1700965.

59. Mithen R, Ho E. Isothiocyanates for human health. Mol Nutr Food Res 2018;62:e1870079.

60. Baird L, Yamamoto M. The molecular mechanisms regulating the KEAP1-NRF2 pathway. Mol Cell Biol 2020;40:e0099-20.

61. Park JE, Sun Y, Lim SK, Tam JP, Dekker M, et al. Dietary phytochemical PEITC restricts tumor development via modulation of epigenetic writers and erasers. Sci Rep 2017;7:40569.

62. Paul B, Li Y, Tollefsbol TO. The effects of combinatorial genistein and sulforaphane in breast tumor inhibition: role in epigenetic regulation. Int J Mol Sci 2018;19:1754.

63. Hyun TK. A recent overview on sulforaphane as a dietary epigenetic modulator. Excli J 2020;19:131-4.

64. Martin SL, Royston KJ, Tollefsbol TO. The role of non-coding RNAs and isothiocyanates in cancer. Mol Nutr Food Res 2018;62:e1700913.

65. Lu Z, Zou J, Li S, Topper MJ, Tao Y, et al. Epigenetic therapy inhibits metastases by disrupting premetastatic niches. Nature 2020;579:284-90.

66. Mitsiogianni M, Amery T, Franco R, Zoumpourlis V, Pappa A, et al. From chemo-prevention to epigenetic regulation: the role of isothiocyanates in skin cancer prevention. Pharmacol Ther 2018;190:187-201.

67. Sehrawat A, Roy R, Pore SK, Hahm ER, Samanta SK, et al. Mitochondrial dysfunction in cancer chemoprevention by phytochemicals from dietary and medicinal plants. Semin Cancer Biol 2017;47:147-53.

68. Sarkar FH, Li YW. Targeting multiple signal pathways by chemopreventive agents for cancer prevention and therapy. Acta Pharmacol Sin 2007;28:1305-15.

69. Bijangi-Vishehsaraei K, Saadatzadeh MR, Wang H, Nguyen A, Kamocka MM, et al. Sulforaphane suppresses the growth of glioblastoma cells, glioblastoma stem cell-like spheroids, and tumor xenografts through multiple cell signaling pathways. J Neurosurg 2017;127:1219-30.

70. Lamy E, Scholtes C, Herz C, Mersch-Sundermann V. Pharmacokinetics and pharmacodynamics of isothiocyanates. Drug Metab Rev 2011;43:387-407.

71. Rakariyatham K, Wu X, Tang Z, Han Y, Wang Q, et al. Synergism between luteolin and sulforaphane in anti-inflammation. Food Funct 2018;9:5115-23.

72. Ibrahim A, Al-Hizab FA, Abushouk AI, Abdel-Daim MM. Nephroprotective effects of benzyl isothiocyanate and resveratrol against cisplatin-induced oxidative stress and inflammation. Front Pharmacol 2018;9:1268.

73. Barrera LN, Cassidy A, Johnson IT, Bao Y, Belshaw NJ. Epigenetic and antioxidant effects of dietary isothiocyanates and selenium: potential implications for cancer chemoprevention. Proc Nutr Soc 2012;71:237-45.

74. Paul B, Li Y, Tollefsbol TO. The effects of combinatorial genistein and sulforaphane in breast tumor inhibition: role in epigenetic regulation. Int J Mol Sci 2018;19:1754.

75. Rakariyatham K, Yang X, Gao Z, Song M, Han Y, et al. Synergistic chemopreventive effect of allylisothiocyanate and sulforaphane on non-small cell lung carcinoma cells. Food Funct 2019;10:893-902. 
76. Jaman MS, Sayeed MA. Ellagic acid, sulforaphane, and ursolic acid in the prevention and therapy of breast cancer: current evidence and future perspectives. Breast Cancer 2018;25:517-28.

77. Agathokleous E, Calabrese EJ. Hormesis: the dose response for the 21st century: the future has arrived. Toxicology 2019;425:152249.

78. Calabrese EJ, Agathokleous E. Building biological shields via hormesis. Trends Pharmacol Sci 2019;40:8-10.

79. Li X, Yang T, Sun Z. Hormesis in health and chronic diseases. Trends Endocrinol Metab 2019;30:944-58.

80. Jodynis-Liebert J, Kujawska M. Biphasic dose-response induced by phytochemicals: experimental evidence. J Clin Med 2020;9:718.

81. Lee YM, Lee DH. Mitochondrial toxins and healthy lifestyle meet at the crossroad of hormesis. Diabetes Metab J 2019;43:568-77.

82. Klaus S, Ost M. Mitochondrial uncoupling and longevity - A role for mitokines? Exp Gerontol 2020;130:110796.

83. Kenny TC, Craig AJ, Villanueva A, Germain D. Mitohormesis primes tumor invasion and metastasis. Cell Rep 2019;27:2292-303.

84. Zelenka J, Koncošová M, Ruml T. Targeting of stress response pathways in the prevention and treatment of cancer. Biotechnol Adv 2018;36:583-602.

85. Tsoupras A, Lordan R, Zabetakis I. Inflammation, not cholesterol, is a cause of chronic disease. Nutrients 2018;10:604.

86. Pein M, Insua-Rodríguez J, Hongu T, Riedel A, Meier J, et al. Metastasis-initiating cells induce and exploit a fibroblast niche to fuel malignant colonization of the lungs. Nat Commun 2020;11:1494.

87. Beckwith JB, Kiviat NB, Bonadio JF. Nephrogenic rests, nephroblastomatosis, and the pathogenesis of Wilms' tumor. Pediat Pathol 1990;10:1-36.

88. Coorens THH, Treger TD, Al-Saadi R, Moore L, Tran MGB, et al. Embryonal precursrs of Wilms tumor. Science 2019;366:1247-51.

89. Thomas ET, Del Mar C, Glasziou P, Wright G, Barratt A, et al. Prevalence of incidental breast cancer and precursor lesions in autopsy studies: a systematic review and meta-analysis. BMC Cancer 2017;17:808.

90. Zill OA, Banks KC, Fairclough SR, Mortimer SA, Vowles JV, et al. The landscape of actionable genomic alterations in cell-free circulating tumor DNA from 21,807 advanced cancer patients. Clin Cancer Res 2018;24:3528-38

91. Sahai E, Astsaturov I, Cukierman E, DeNardo DG, Egeblad M, et al. A framework for advancing our understanding of cancer- associated fibroblasts. Nature Rev Cancer 2020;20:174-86.

92. Monteran L, Erez N. The dark side of fibroblasts: cancer-associated fibroblasts as mediators of immunosuppression in the tumor microenvironment. Front Immunol 2019;10:1835.

93. Rothlin, CV, Ghosh S. Lifting the innate immune barriers to antitumor immunity. J Immunother Cancer 2020;8:e00695.

94. Sturm C, Wagner AE. Brassica-derived plant bioactives as modulators of chemopreventive and inflammatory signaling pathways. Int J Mol Sci 2017;18:1890.

95. Liang J, Jahraus B, Balta E, Ziegler JD, Hübner K, et al. Sulforaphane inhibits inflammatory responses of primary human t-cells by increasing ROS and depleting glutathione. Front Immunol 2018;9:2584.

96. Burčul F, Generalić Mekinić I, Radan M, Rollin P, Blažević I. Isothiocyanates: cholinesterase inhibiting, antioxidant, and antiinflammatory activity. J Enzyme Inhib Med Chem 2018;33:577-82.

97. Yoo IH, Kim MJ, Kim J, Sung JJ, Park ST, et al. The anti-inflammatory effect of sulforaphane in mice with experimental autoimmune encephalomyelitis. J Korean Med Sci 2019;34:e197.

98. Wang Y, Lu J, Jiang B, Guo J. The roles of curcumin in regulating the tumor immunosuppressive microenvironment. Oncol Lett 2020;19:3059-70.

99. Marrazzo P, Angeloni C, Hrelia S. Combined treatment with three natural antioxidants enhances neuroprotection in a SH-SY5Y 3D culture model. Antioxidants (Basel) 2019;8:420.

100. Grafetstätter M, Pletsch-Borba L, Sookthai D, Karavasiloglou N, Johnson T, et al. Thrombomodulin and thrombopoietin, two biomarkers of hemostasis, are positively associated with adherence to the world cancer research fund/american institute for cancer research recommendations for cancer prevention in a population-based cross-sectional study. Nutrients 2019;11:2067.

101. Uppal S, Kaur K, Kumar R, Kaur ND, Shukla G, et al. Chitosan nanoparticles as a biocompatible and efficient nanowagon for benzyl isothiocyanate. Int J Biol Macromol 2018;115:18-28.

102. Nasery M, Abadi B, Poormoghadam D, Zarrabi A, Keyhanvar P, et al. Curcumin delivery mediated by bio-based nanoparticles: a review. Molecules 2020;25:689.

103. Liu P, Behray M, Wang Q, Wang W, Zhou Z, et al. Anti-cancer activities of allyl isothiocyanate and its conjugated silicon quantum dots. Sci Rep 2018;8:1084.

104. Lane AN, Higashi RM, Fan TW. Metabolic reprogramming in tumors: contributions of the tumor microenvironment. Genes Dis 2020;7:185-98.

105. Piskovatska V, Stefanyshyn N, Storey KB, Vaiserman AM, Lushchak O. Metformin as a geroprotector: experimental and clinical evidence. Biogerontology 2019;20:33-48.

106. Kwon Y. Food-derived polyphenols inhibit the growth of ovarian cancer cells irrespective of their ability to induce antioxidant responses. Heliyon 2018;4:e00753.

107. Lăcătușu CM, Grigorescu ED, Floria M, Onofriescu A, Mihai BM. The mediterranean diet: From an environment-driven food culture to an emerging medical prescription. Int J Environ Res Public Health 2019;16:942.

108. D'Innocenzo S, Biagi C, Lanari M. Obesity and the mediterranean diet: a review of evidence of the role and sustainability of the mediterranean diet. Nutrients 2019;11:1306.

109. Uusitupa M, Khan TA, Viguiliouk E, Kahleova H, Rivellese AA, et al. Prevention of type 2 diabetes by lifestyle changes: a systematic review and meta-analysis. Nutrients 2019;11:2611.

110. Yagishita Y, Fahey JW, Dinkova-Kostova AT, Kensler TW. Broccoli or sulforaphane: Is it the source or dose that matters? Molecules 
2019;24:3593.

111. Quirante-Moya S, García-Ibañez P, Quirante-Moya F, Villaño D, Moreno DA. The role of brassica bioactives on human health: are we studying it the right way? Molecules 2020;25:1591.

112. Phan MAT, Paterson J, Bucknall M, Arcot J. Interactions between phytochemicals from fruits and vegetables: effects on bioactivities and bioavailability. Crit Rev Food Sci Nutr 2018;58:1310-29.

113. Romeo L, Iori R, Rollin P, Bramanti P, Mazzon E. Isothiocyanates: an overview of their antimicrobial activity against human infections. Molecules 2018;23:624.

114. Pedersen BK. Regular exercise helps patients combat cancer. Scientist 2020. Available from: https://www.the-scientist.com/features/ regular-exercise-helps-patients-combat-cancer-67317. [Last accessed on 29 Jul 2020]

115. Musci RV, Hamilton KL, Linden MA. Exercise-induced mitohormesis for the maintenance of skeletal muscle and healthspan extension. Sports (Basel) 2019;7:170. 